Proceedings of the International School and Conference on Optics and Optical Materials, ISCOM07, Belgrade, Serbia, September 3-7, 2007

\title{
Imaging Properties of Laser-Produced Parabolic Profile Microlenses
}

\author{
D. Vasiluevićc , B. Murić, D. Pantelić and B. Panić \\ Institute of Physics, Pregrevica 118, 11080 Belgrade, Serbia \\ Imaging properties of the concave microlens are presented. Microlenses \\ are produced by using tot'hema eosin sensitized gelatin and $532 \mathrm{~nm}$ laser ir- \\ radiation (2nd Nd:YAG harmonic). Imaging properties of microlenses are \\ analized by calculation: the RMS wave front aberration, the diffraction \\ point spread function cross section and the spot diagram. The obtained \\ microlenses had excellent, near diffraction limited, performance for the mod- \\ erate field angles.
}

PACS numbers: 42.70.Gi, 81.05.Zx, 42.30.Lr, 42.15.Fr

\section{Introduction}

Production and application of microoptical elements is a new and fast expanding field in optics. Microoptical elements can be used in various applications like wave front sensing, diffusers, integral optics, Luneburg and geodesic lenses, arrays and waveguides [1-4]. One of the most important components in microoptical elements are microlenses. Like classical lens, microlens can be a convex or a concave lens and can have a spherical or an aspherical profile $[5,6]$. Usually microlenses have dimensions from several tens of micrometers to one millimetre. Microlenses are usually produced by one of the following methods: mechanical copying or embossing, melting, photolithography, direct laser writing [7-10]. Diverse materials like photosensitive glasses, organic and biological polymers, composites and liquid crystals can be used for fabrication of microlenses [11-15].

In this paper imaging properties of microlenses produced on a layer of tot'hema and eosin sensitized gelatin (TESG), by focused beam Nd:YAG laser operating at $532 \mathrm{~nm}$ is presented. Tot'hema is a drinkable solution used in medicine for curing an iron deficit in human organism. Eosin is an organic dye, with absorption maximum in the green part of the spectrum used in medicine, too. Chemical components are well known, easily accessible, nonpoisonous and cheap. Five different microlenses were produced by placing TESG layer at the focus of the laser

*corresponding author; e-mail: darko@phy.bg.ac.yu 
beam and at distances of $1 \mathrm{~cm}, 2 \mathrm{~cm}, 3 \mathrm{~cm}$ and $4 \mathrm{~cm}$ from the focus. The obtained microlenses had excellent, near diffraction limited, performances for the moderate total field of view $\left(2 \omega=16^{\circ}\right)$. Imaging properties are analyzed by: the root mean squares (RMS) wave front aberration, the diffraction point spread function (PSF) cross-section, and the spot diagram.

\section{Microlens fabrication}

Fabrication process of microlenses begins with the preparation of the TESG layer on a microscope glass substrate, by the gravity settling method (the procedure outlined in Table I). Detailed description of the fabrication process is given in $[16]$.

TABLE I

Procedure for preparing TESG layer.

\begin{tabular}{c|l}
\hline \hline Step & \multicolumn{1}{|c}{ Description } \\
\hline 1 & $\begin{array}{l}\text { Swell of } 0.5 \mathrm{~g} \text { commercial-quality gelatin (edible gelatin) in } 10 \mathrm{ml} \text { deionised } \\
\text { water for } 60 \mathrm{~min} .\end{array}$ \\
\hline 2 & Heat the suspension in a thermostated water bath at $50^{\circ} \mathrm{C}$, with stirring. \\
\hline 3 & Add $2 \mathrm{ml}$ of solution of tot'hema with stirring. \\
\hline 4 & Add $0.03 \mathrm{ml}$ of $1 \%$ aqueous solution of eosin with stirring. \\
\hline 5 & Centrifuge TESG solution in order to remove impurities. \\
\hline 6 & $\begin{array}{l}\text { Pipette out of } 1 \mathrm{ml} \text { of TESG solution onto a precisely levelled and well-cleaned } \\
\text { microscope glass substrate. }\end{array}$ \\
\hline 7 & $\begin{array}{l}\text { Dry the plate in the dark, overnight, in relatively stable environmental condi- } \\
\text { tions. }\end{array}$
\end{tabular}

The dried TESG layer thickness of $100 \mu \mathrm{m}$ was obtained and exposed with focused Nd:YAG laser beam operating at $532 \mathrm{~nm}$. During irradiation, a series of two systems of bright and dark concentric rings was produced on the screen, placed behind the sample. The number and diameter of these rings increase over time, reaching steady state after a few seconds (the lens is completely formed). Further, produced TESG lenses were processed in isopropyl bath and after that they were stable, without degradation.

\section{Geometry of microlenses}

The profiles of microlenses were measured by Talystep ${ }^{\text {TM }}$ surface profiler manufactured by Taylor-Hobson Ltd. Obtained results are discussed in [16, 17] and they show that produced microlenses are concave lenses with parabolic profiles in the central part ( $80 \%$ of the aperture).

In this paper five concave microlenses with parabolic profile are discussed. They are produced by placing TESG layer at the focus of the laser beam and at 
distances of $1 \mathrm{~cm}, 2 \mathrm{~cm}, 3 \mathrm{~cm}$ and $4 \mathrm{~cm}$ from the focus. The parabolic profile of microlenses can be defined for the purpose of raytracing by the even asphere surface. The general definition for the even asphere surface is

$$
\begin{aligned}
z= & \frac{c r^{2}}{1+\sqrt{1-(1+k) c^{2} r^{2}}}+\alpha_{1} r^{2}+\alpha_{2} r^{4}+\alpha_{3} r^{6}+\alpha_{4} r^{8}+\alpha_{5} r^{10} \\
& +\alpha_{6} r^{12}+\alpha_{7} r^{14}+\alpha_{8} r^{16}
\end{aligned}
$$

where $c$ - the curvature (the reciprocal of the radius), $r=\sqrt{x^{2}+y^{2}}$ - the aperture of the lens (the radial coordinate), $k$ - the conic constant, $\alpha_{1}-\alpha_{8}-$ the polynomial coefficients. For given parabolic profile of microlens equation for even asphere surface is reduced to

$$
z=\alpha_{1} r^{2}
$$

where $\alpha_{1}$ is the coefficient of parabola. Table II.

The basic geometrical parameters for five produced microlenses are given in

TABLE II

Basic geometrical parameters for produced concave microlenses $\mathrm{M}$.

\begin{tabular}{l|c|c|c|c|c}
\hline \hline & M1 & M2 & M3 & M4 & M5 \\
\hline coefficient of parabola [-] & -17 & -12 & -6 & -4 & -3 \\
lens thickness [mm] & 0.078 & 0.082 & 0.088 & 0.094 & 0.097 \\
effective focal length [mm] & -0.055 & -0.078 & -0.155 & -0.230 & -0.310 \\
numerical aperture [-] & 0.48 & 0.36 & 0.19 & 0.13 & 0.10
\end{tabular}

The clear aperture of all produced concave microlenses is $0.06 \mathrm{~mm}$. The index of refraction for TESG layer $(n=1.537)$ is determined in our research of imaging properties of microlens produced by unfocused laser beam published in $[17]$.

\section{Imaging properties of microlenses}

Imaging properties of presented concave microlenses are calculated by raytracing. In order to determine image quality of microlenses moderate total field of view $\left(2 \omega=16^{\circ}\right)$ is used. Imaging properties of microlenses are characterized through: the RMS wave front aberration, the diffraction point spread function cross-section, and the spot diagram. Those are standard means of determination of image quality when aberrations are small and optical system is the diffraction limited system.

Before starting any image analysis it is important to mention that five concave microlenses that are analysed are quite different lenses ranging from relatively small effective focal length and large $F$ number $(F / 1)$ to relatively large effective focal length and small $F$ number $(F / 5)$. 
The wave front aberration is usually used when aberrations are small and can be compared with diffraction. The RMS wave front aberration vs. the field of view for five concave microlenses is presented in Fig. 1.

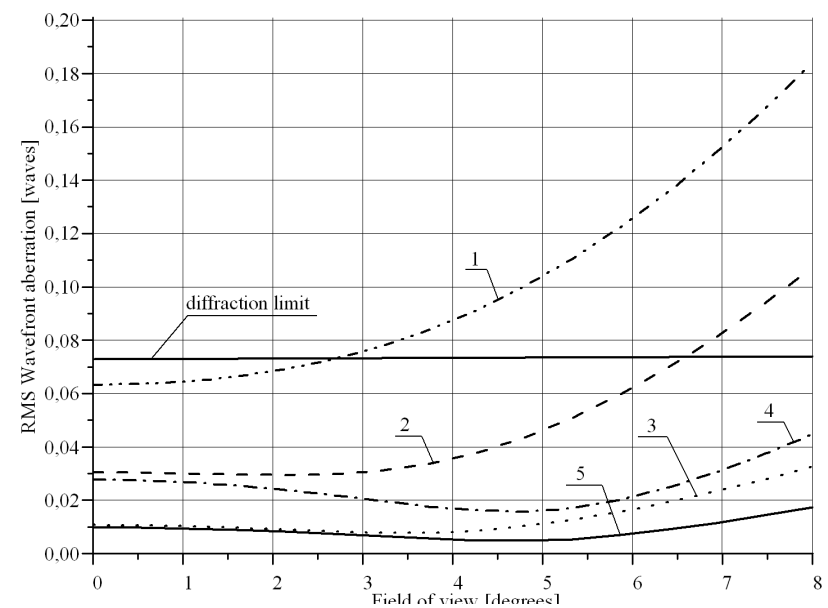

Fig. 1. The RMS wave front aberration. $1-$ microlens $1,2-$ microlens $2,3-$ microlens 3, 4 - microlens 4, 5 - microlens 5 .

From Fig. 1 one can see that all microlenses have the diffraction limited performance or near diffraction limited performance. If the wave front aberration is smaller than diffraction then the diffraction limits performances of optical system, for the optical system with smaller aberrations than the diffraction is said to be diffraction limited system. Goal of each optical designer is to try to design the diffraction limited system. All presented microlenses can be divided into two groups. The first group consists of microlens 1 and 2 which have smaller effective focal lengths $(-0.055 \mathrm{~mm}$ and $-0.078 \mathrm{~mm})$ and larger numerical apertures $(0.48$ and 0.36 , corresponding $F$ numbers are $F / 1$ and $F / 1.3)$. The second group consists of microlens 3,4 , and 5 which have larger effective focal lengths $(-0.155 \mathrm{~mm}$, $-0.230 \mathrm{~mm}$, and $-0.310 \mathrm{~mm})$ and smaller numerical apertures $(0.19,0.13$, and 0.10 , corresponding $F$ numbers are $F / 3, F / 4$, and $F / 5)$. It is well known that aberrations among other factors depend on the numerical aperture (the larger numerical aperture the larger aberrations will be). The second group of microlenses have the diffraction limited performance because the RMS wave front aberration is smaller than diffraction for all angles in field of view. The first group of microlenses have near diffraction limited performance because it has smaller RMS wave front aberration than diffraction for smaller angles in field of view and larger RMS wave front aberration than diffraction for larger angles in field of view.

Next imaging property of microlens that is analysed is the diffraction PSF cross-section. The PSF is obtained when fast Fourier transform (FFT) algorithm is applied to the difference of the wave front aberration (a real optical system) 


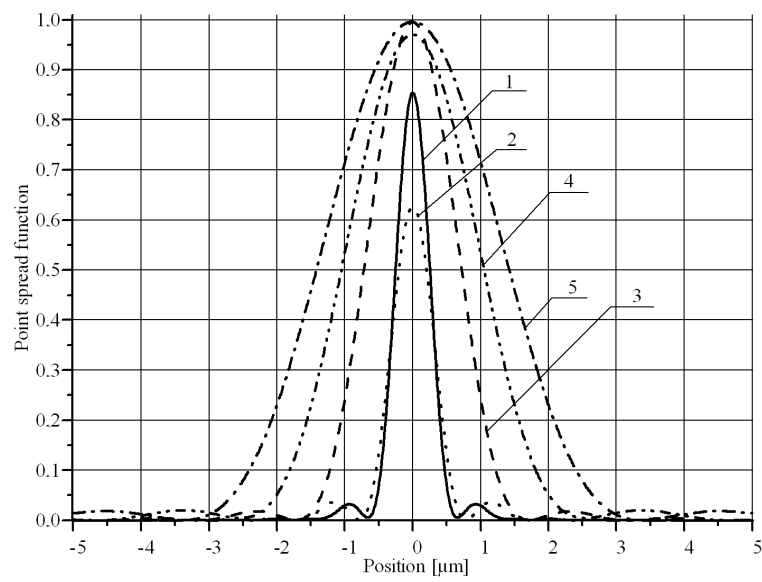

Fig. 2. The diffraction PSF cross-section. 1 - microlens 1, 2 - microlens 2, 3 microlens 3, 4 - microlens 4, 5 - microlens 5 .

and the reference sphere (an ideal optical system) at the exit pupil. The PSF cross-section for five concave microlenses is presented in Fig. 2.

The PSF can be considered as the representation of illumination intensity of point object. If aberrations of an optical system are small then the illumination intensity of point object of real optical system is close to one of ideal optical system. Two groups of microlenses can be seen by analysing PSF cross-sections of presented concave microlenses. The first group consists of microlenses 1 and 2 which have larger numerical aperture and larger wave front aberrations and because of that smaller PSF. The second group consists of microlenses 3, 4, and 5 which have smaller numerical aperture and smaller wave front aberrations and because of that larger PSF.

The spot diagram represents method for geometrical analysis of point object image quality. It is calculated by tracing large number of rays (hundreds or thousands) through an entrance pupil and an optical system. For each ray, intersection with the image plane is calculated. The spot diagram can be represented by the RMS spot radius and the geometrical spot radius. The geometrical spot radius takes in account every ray that is passed through the optical system and it is defined as a simple distance between the reference point and the most distant ray. The RMS spot radius uses special algorithm for calculation of spot radius. In order to make decision about the image quality of optical system the RMS spot radius is compared with the Airy disc radius. The Airy disc radius is the radius to the first dark ring of the Airy disc for a circular, uniformly illuminated entrance pupil. The necessary data for spot size analysis for presented concave microlenses is given in Table III.

The presented data for the RMS spot radius and the geometrical spot radius is for the maximum field angle $\left(\omega=8^{\circ}\right)$. It can be seen that microlenses 2 to 
TABLE III

Spot size parameters for presented concave microlenses M.

\begin{tabular}{l|c|c|c|c|c}
\hline \hline & M1 & M2 & M3 & M4 & M5 \\
\hline Airy disc radius $[\mu \mathrm{m}]$ & 0.688 & 0.910 & 1.716 & 2.543 & 3.376 \\
RMS spot radius $[\mu \mathrm{m}]$ & 1.085 & 0.682 & 0.446 & 0.959 & 0.402 \\
Geometrical spot radius $[\mu \mathrm{m}]$ & 2.137 & 1.274 & 0.739 & 2.741 & 0.767
\end{tabular}

5 are diffraction limited optical systems because they have the RMS spot radius smaller than the Airy disc radius. The microlens 1 has large numerical aperture ( $N A=0.48$ and corresponding $F$ number is $F / 1)$. It is very hard to have aberrations smaller than diffraction when you have large numerical aperture and moderate field of view.

\section{Conclusions}

In this paper there are described concave microlenses produced using tot'hema eosin sensitized gelatin and $532 \mathrm{~nm}$ laser irradiation. Imaging properties of microlenses are analyzed by calculation: the RMS wave front aberration, the diffraction point spread function cross-section, and the spot diagram. Five presented microlenses can be divided into two categories: the first with smaller effective focal lengths and larger numerical apertures (microlenses 1 and 2) which have near diffraction limited performance and the second with larger effective focal lengths and smaller numerical apertures (microlenses $3,4,5$ ) which have diffraction limited performance.

\section{Acknowledgments}

This paper was written with the support of the Ministry of Science and Environmental Protection of the Republic of Serbia, under the project No. 141003.

\section{References}

[1] Ph. Nussbaum, R. Völkely, H.P. Herzig, M. Eisner, S. Haselbeck, Pure Appl. Opt. 6, 617 (1997).

[2] T.R.M. Sales, S. Chakmakjian, G.M. Morris, D.J. Schertler, Photonics Spectra 38, 58 (2004).

[3] J.R. Flores, J. Sochacki, Appl. Opt. 33, 3409 (1994).

[4] S. Calixto, G. Paez Padilla, Appl. Opt. 35, 6126 (1996).

[5] D.W. de Lima Monteiro, O. Akhzar-Mehr, P.M. Sarro, G. Vdovin, Opt. Express 11, 2244 (2003).

[6] R. Grunwald, S. Woggon, R. Ehlert, W. Reinecke, Pure Appl. Opt. 6, 663 (1997).

[7] Y. Fu, N.K. Bryan, IEEE Trans. Semicond. Manufact. 15, 229 (2002).

[8] R. Grunwald, H. Mischke, W. Rehak, Appl. Opt. 38, 4117 (1999). 
[9] M. Wakaki, Y. Komachi, G. Kanai, Appl. Opt. 37, 627 (1998).

[10] M.-H. Wu, C. Park, G.M. Whitesides, Langmuir 18, 9312 (2002).

[11] M. He, X.-C. Yuan, N.Q. Ngo, J. Bu, S.H. Tao, J. Opt. A, Pure Appl. Opt. 6, 94 (2004).

[12] S. Calixto, M.S. Scholl, Appl. Opt. 36, 2101 (1997).

[13] S. Mihailov, S. Lazare, Appl. Opt. 32, 6211 (1993).

[14] C.D. Jones, M.J. Serpe, L. Schroeder, L.A. Lyon, J. Am. Chem. Soc. 125, 5292 (2003).

[15] S. Masuda, S. Takahashi, T. Nose, S. Sato, H. Ito, Appl. Opt. 30, 4772 (1997).

[16] B. Murić, D. Pantelić, D. Vasiljević, B. Panić, Opt. Mater., 2007, doi:10.1016/j.optmat.2007.05.051.

[17] D. Vasiljević, D. Pantelić, B. Murić, in: 14th Int. School on Quantum Electronics: Laser Physics and Applications, Proceeding of SPIE, Vol. 6604, paper no. 66040Q, Eds. P. Atanasov, T. Dreishchuh, S. Gatova, International Society for Optical Engineering, Bellingham (WA) 2007. 